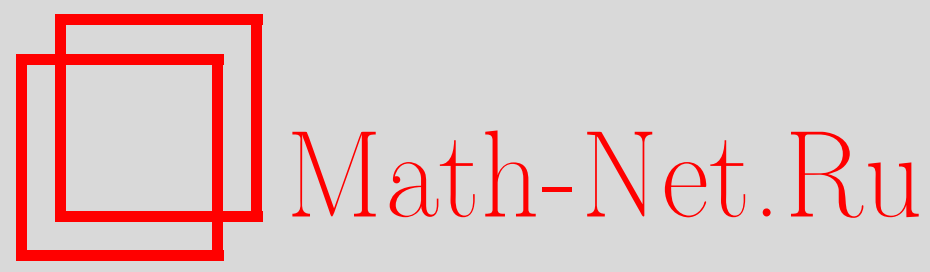

И. В. Яковлев, Модельный подход к нестандартному анализу в рамках аксиоматической теории множеств, Матем. заметки, 2006, том 79, выпуск 1, 134-141

DOI: https://doi.org/10.4213/mzm2675

Использование Общероссийского математического портала Math-Net.Ru подразумевает, что вы прочитали и согласны с пользовательским соглашением http: //www . mathnet.ru/rus/agreement

Параметры загрузки:

IP: 3.93 .64 .190

26 апреля 2023 г., $18: 37: 12$

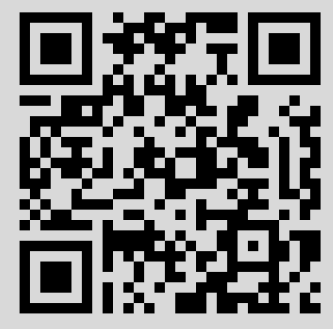




\section{МОДЕЛЬНЫЙ ПОДХОД К НЕСТАНДАРТНОМУ АНАЛИЗУ В РАМКАХ АКСИОМАТИЧЕСКОЙ ТЕОРИИ МНОЖЕСТВ}

\section{И.В. Яковлев}

В данной заметке модельный подход к нестандартному анализу развит на основе аксиоматической теории множеств Цермело-Френкеля с атомами. Дано обоснование традиционного рассмотрения стандартной суперстуктуры $V$ в качестве первичного объекта нестандартного анализа. Получены теоретико-множественные аксиомы для нестандартной системы $* V$.

Библиографояи: 9 названий.

Введение. Теория нестандартных методов анализа использует два альтернативных подхода: модельный и аксиоматический. Модельньй подход (МП) к нестандартному анализу [1]-[3] устанавливает с помощью метода ультрапроизведений ряд фактов, которые принимаются в качестве аксиом нестандартной теории множеств [4], [5], однако сам по себе он излагается с позиций наивной теории множеств. При этом характерной чертой МП служит наличие бесконечного множества индивидов, или атомов - объектов, которые не содержат элементов и множествами не являются. Идея использования теоретико-множественной аксиоматики, включающей атомы, в качестве основы МП была высказана В. Ф. Яковлевым [6]. Настоящая заметкапосвящена реализации МП в рамках теории множеств Цермело-Френкеля с атомами (ZFCA).

Основным объектом МП является суперструктура

$$
V=\bigcup_{n=0}^{\infty} V_{n}
$$

над множеством атомов $V_{0}[1]$. В традиционной схеме изложения МП суперструктура $V$ рассматривается как первичный и, в некотором смысле, "максимальный" объект теории. Так, в качестве множеств фигурируют лишь элементы $V$, отличные от атомов, и отношение $\epsilon$ определяется только на элементах $V$. С точки зрения ZFCA, однако, суперструктура является собственной частью универсума всех атомов и множеств. Тем не менее, приводимые в данной заметке построения обосновьвают принятие именно ее в качестве стандартной алгебраической системы для формального языка ZFCA. B peзультате ограничения "большого" универсума не все аксиомы ZFCA будут истинны в полученной системе; аксиомы, остающиеся истинньми, переносятся в нестандартную систему $* V$ и составляют там базовьй набор утверждений о внутренних атомах и множествах. Абсолютность основных теоретико-множественных формул и операций позволяет единьм образом доказать все наиболее важные свойства нестандартных образов множеств, отношений и функций. 
1. Теория ZFCA. Алфавит языка ZFCA есть алфавит языка ZFC, дополненный константой $A$ для множества атомов. С целью сокращения записи формул мы используем прописные латинские буквы $X, Y$ для имен только множеств. Так, для формулы $\varphi(x)$

$$
\forall X \varphi(X) \leftrightharpoons \forall x(x \notin A \rightarrow \varphi(x)), \quad \exists X \varphi(X) \leftrightharpoons \exists x(x \notin A \wedge \varphi(x))
$$

(запись слева от знака е есть обозначение для выражения справа).

Вводятся классы $\mathbb{A} \leftrightharpoons\{x \mid x=x\}$ (универсум всех атомов и множеств), $\mathbb{M} \leftrightharpoons\{x \mid$ $x \notin A\}$ (класс множеств), $\varnothing \leftrightharpoons\{x \mid x \neq x\}$. Теоретико-множественные операции определяются так же, как в [7]. Отметим, что

$$
y \subset x \leftrightharpoons x \in \mathbb{M} \wedge y \in \mathbb{M} \wedge \forall z \in y(z \in x)
$$

Ниже следуют аксиомы ZFCA. Некоторые аксиомы приводимого списка могут быть выведены из остальных; вопрос о независимости системы аксиом не является в данном случае принципиальньп.

(А0) Аксиома Атомов [7]. $\forall x(x \in A \leftrightarrow x \neq \varnothing \wedge \forall y(y \notin x))$.

Отсюда $\varnothing \in \mathbb{M}$ и $x \in \mathbb{M} \wedge \forall y(y \notin x) \rightarrow x=\varnothing$.

(A1) АКСиома оБъЕмности. $\forall X \forall Y(X \subset Y \wedge Y \subset X \rightarrow X=Y)$.

Равенство атомов интерпретируется как совпадение элементов множества, являющегося интерпретацией константы $A$.

(А2) АкСИомА ПАРЫ. $\forall x \forall y(\{x, y\} \in \mathbb{M})$.

(А3) АКСИомА ВЫДЕЛЕНИЯ (СХемА). $\forall \vec{y} \forall X(\{x \in X \mid \varphi(x, \vec{y})\} \in \mathbb{M})$.

Здесь используются обозначения, принятые в [7]. Подстановка вместо $X$ имени атома дает истинное $\varnothing \in \mathbb{M}$. Данная аксиома влечет $x \cap y \in \mathbb{M}$ и $x \backslash y \in \mathbb{M}$ для всех $x, y$.

(А4) АКСИОМА ОБЪЕДИНЕНИЯ. $\forall X(\cup X \in \mathbb{M})$.

При $x \in A$ имеем $\cup x=\varnothing \in \mathbb{M}$.

(A5) АКСИОМА СТЕПЕНИ. $\forall X(\mathscr{P}(X) \in \mathbb{M})$.

При $x \in A$ имеем $\mathscr{P}(x)=\varnothing \in \mathbb{M}$.

(А6) АКСИОМА ПоДСТАНОВКИ (СХемА). $\forall \vec{z}(\forall x \exists ! y \varphi(x, y, \vec{z}) \rightarrow \forall X(\{y \mid \exists x \in$ $X \varphi(x, y, \vec{z})\} \in \mathbb{M}))$.

(А7) АКСИОМА БЕСКонЕчности. $\exists X(\varnothing \in X \wedge \forall x \in X(x \cup\{x\} \in X))$.

(А8) АКСИома РЕГУлЯРности. $\forall X \neq \varnothing \exists x \in X(x \cap X=\varnothing)$.

Всякая $\in$-убьвающая последовательность обрывается на пустом множестве или атомe. 
(А9) Аксиома выбора. $\forall X(X \neq \varnothing \wedge X \cap A=\varnothing \wedge \forall Y \in X(Y \neq \varnothing) \rightarrow \exists f(f-$ функция выбора на $X)$ ).

Универсум ZFCA имеет вид

$$
\mathbb{A}=\bigcup_{\alpha \in \mathrm{On}} A_{\alpha}, \quad A_{0}=A, \quad A_{\alpha+1}=A_{\alpha} \cup \mathscr{P}\left(A_{\alpha}\right), \quad A_{\lambda}=\bigcup_{\alpha<\lambda} A_{\alpha}
$$

(On - класс всех ординалов, $\lambda$ всегда обозначает бесконечный предельный ординал). Каждое $A_{\alpha}$ транзитивно [7]. Для всякого $x$ его ранг $\operatorname{rank}(x)$ есть наименьший ординал $\alpha$ такой, что $x \in A_{\alpha}$.

2. Ультрастепень. Стандартной интерпретацией константы $A$ служит бесконечное множество $S$, конкретный выбор которого определяется областью приложений нестандартных методов. Тогда в соответствии с (1) получаем стандартный универсум

$$
\mathbb{V}=\bigcup_{\alpha \in \mathrm{On}} V_{\alpha}, \quad V_{0}=S, \quad V_{\alpha+1}=V_{\alpha} \cup \mathscr{P}\left(V_{\alpha}\right), \quad V_{\lambda}=\bigcup_{\alpha<\lambda} V_{\alpha}
$$

и стандартную суперструктуру, которая отвечает первому бесконечному ординалу $\omega$ :

$$
V \leftrightharpoons V_{\omega}=\bigcup_{n \in \omega} V_{n}
$$

Посредством $a, \vec{a}, b, \vec{b}$ всегда обозначаются элементы $V$.

Пусть $\mathscr{D}$ - счетно-неполньй ультрафильтр на бесконечном множестве $I$ (счетная неполнота ультрафильтра обеспечивает насыщенность ультрастепени [8], что необходимо для приложений нестандартного анализа). При $f, g \in \mathbb{V}^{I}$ полагаем [7]:

$$
\begin{aligned}
& f \sim g \leftrightharpoons f_{i}=g_{i} \text { п.в. }\left\{i \in I \mid f_{i}=g_{i}\right\} \in \mathscr{D}, \\
& {[f] \leftrightharpoons\{u \mid u \sim f \wedge \forall v(v \sim f \rightarrow \operatorname{rank}(u) \leqslant \operatorname{rank}(v))\},} \\
& \mathbb{U} \leftrightharpoons\left\{[f] \mid f \in \mathbb{V}^{I}\right\}, \\
& {[f] \in \mathscr{D}[g] \leftrightharpoons f_{i} \in g_{i} \text { п.в. }}
\end{aligned}
$$

Пусть $U_{\alpha} \leftrightharpoons\left\{[f] \mid f_{i} \in V_{\alpha}\right.$ п.в. $\} ; U_{\alpha} \subset U_{\beta}$ при $\alpha<\beta$. Так как всякая функция $f \in \mathbb{V}^{I}$ является множеством, то

$$
\mathbb{U}=\bigcup_{\alpha \in \mathrm{On}} U_{\alpha}
$$

В частности,

$$
U \leftrightharpoons \bigcup_{n \in \omega} U_{n}
$$

- ограниченная ультрастепень суперструктуры $V$. Вследствие счетной неполноты ультрафильтра строение ультрастепени отличается от теоретико-множественного универсума.

УТВЕРЖДЕНИЕ 1. (i) $U_{\omega} \backslash U \neq \varnothing$.

(ii) Ультрастепень содержит бесконечную $\in \mathscr{D}-$ убвваючу последовательность. 
ДокАЗАТЕЛьСтво. Поскольку $\mathscr{D}$ счетно-неполон, существуют попарно непересекаюшиеся множества $E_{n} \subset I$ такие, что $E_{n} \notin \mathscr{D}$ для всех $n \in \omega$ и

$$
\bigcup_{n \in \omega} E_{n}=I
$$

Положим

$$
a_{0} \in S, \quad a_{n+1}=\left\{a_{n}\right\}
$$

и определим функцию $g$ на $I$ следуюшим образом:

$$
g_{i}=a_{n} \quad \text { при } i \in E_{n} .
$$

Тогда $g_{i} \in V_{\omega}$ для всех $i \in I$, однако неверно, что $g_{i} \in V_{n}$ п.в. для некоторого $n$. Следовательно, $[g] \in U_{\omega} \backslash U$, что доказывает (i). Для доказательства (ii) определим оператор $R$, действующий на построенную функцию $g$ :

$$
(R g)_{i}=\left\{\begin{array}{l}
a_{0}, \text { если } i \in E_{0} \\
a_{n}, \text { если } i \in E_{n+1}
\end{array}\right.
$$

Тогда $[g] \ni \mathscr{D}[R g] \ni \mathscr{D}\left[R^{2} g\right] \ni \mathscr{D} \ldots$. Утверждение доказано.

3. Нестандартная система. Полагая в качестве (нестандартной) интерпретации константы $A$ множество $U_{0}$, получим нестандартньй универсум

$$
\mathbb{W}=\bigcup_{\alpha \in \mathrm{On}} W_{\alpha}, \quad W_{0}=U_{0}, \quad W_{\alpha+1}=W_{\alpha} \cup \mathscr{P}\left(W_{\alpha}\right), \quad W_{\lambda}=\bigcup_{\alpha<\lambda} W_{\alpha}
$$

и соответствующую суперструктуру

$$
W \leftrightharpoons W_{\omega}=\bigcup_{n \in \omega} W_{n}
$$

Поуровневое изоморфное вложение $*: U \rightarrow W$ дается функцией Мостовского:

* тождественна на $U_{0}$,

$$
* f \leftrightharpoons *[f]=\{* u \mid[u] \in \mathscr{D}[f]\} \text { при }[f] \in U \backslash U_{0}
$$

ввиду тождественности на атомах этот изоморфизм является единственным [9]. Образ $U$ есть нестандартная система $* V=\{* f \mid[f] \in U\}$. Посредством $* a, * \vec{a}, * b, * \vec{b}$ всегда обозначаются стандартные элементы $* V$, построенные из соответствующих элементов $V$.

Факты, отмеченные в утверждении 1 , не позволяют продолжить *-отображение за уровень $\omega$.

УТВЕРЖДЕНИЕ 2. Не существует изоморфного вложсения $U_{\omega}$ в $\mathbb{W}$. 
ДокАЗАТЕЛЬСТво. Используем $\left\{a_{n}\right\}_{n \in \omega}, g$ и $R$, определенныев (2)-(4). Определим также оператор $T$ :

$$
(T g)_{i}=a_{n+1} \quad \text { при } i \in E_{n} .
$$

Тогда $\left[T^{m} R^{m} g\right]=[g]$ для всякого натурального $m$. Предположим, что существует изоморфное вложение о $: U_{\omega} \rightarrow \mathbb{W}$ (вместо о $[f]$ пишем of). Очевидно, о $g \notin W_{n}$ при всех $n \in \omega ;$ значит, $\operatorname{rank}(\circ g)=\lambda+m$ для некоторого натурального $m \neq 0$. Тогда $\circ\left(R^{m} g\right) \in W_{\lambda}$, т.е. $\circ\left(R^{m} g\right) \in W_{\alpha}$ для некоторого $\alpha<\lambda$, и $\circ=\circ\left(T^{m} R^{m} g\right) \in W_{\alpha+m}-$ в противоречии с определением ранга, ибо $\alpha+m<\lambda+m$. Утверждение доказано.

Таким образом, изоморфное отображение из $\mathbb{U}$ в $\mathbb{W}$ можно определить лишь до уровня $U_{\omega}$, т.е. лишш для ограниченной ультрастепени $U$. Данное обстоятельство и вьнуждает с самого начала рассматривать в качестве стандартной системы суперструктуру $V$. Одновременно возникает требование ограниченности кванторов (действие каждого квантора в формуле должно быть ограничено множеством - элементом суперструктуры $\left.A_{\omega}\right)$, которое необходимо для доказательства “ограниченного” варианта теоремы Лося [1] и, как следствие, принципа переноса:

$$
V \models \varphi(\vec{a}) \leftrightarrow * V \models \varphi(* \vec{a})
$$

для формулы $\varphi(\vec{x})$ с ограниченными кванторами.

Условие ограниченности кванторов можно частично ослабить в следующем случае. Пусть $\varphi(\vec{x}, \vec{y})$ - формула с ограниченными кванторами.

УТВЕРЖДЕНИЕ 3. Имеем $V \models \forall \vec{x} \varphi(\vec{x}, \vec{b}) \leftrightarrow * V \models \forall \vec{x} \varphi(\vec{x}, * \vec{b})$.

ДокаЗАТЕЛЬСтво. Пусть $V \models \varphi(\vec{a}, \vec{b})$ для всякого $\vec{a}$. Возьмем произвольное $* \vec{f}$. Тогда $V=\varphi\left(\vec{f}_{i}, \vec{b}\right)$ для всех $i \in I$. По теореме Лося имеем $* V \models \varphi(* \vec{f}, * \vec{b})$. Обратно, пусть $* V \models \varphi(* \vec{f}, * \vec{b})$ для всех $* \vec{f}$. Тогда для любого $\vec{a}$ истинно $* V \models \varphi(* \vec{a}, * \vec{b})$ и, следовательно, $V \models \varphi(\vec{a}, \vec{b})$. Утверждение доказано.

СледСТВиЕ 1. Имеем $V \models \exists \vec{x} \varphi(\vec{x}, \vec{b}) \leftrightarrow * V \models \exists \vec{x} \varphi(\vec{x}, * \vec{b})$.

СлЕДСТВИЕ 2. Если $* V \models \varphi(* \vec{a}, * \vec{b})$ для любого стандартного $* \vec{a}$, mо $* V \models$ $\forall \vec{x} \varphi(\vec{x}, * \vec{b})$.

Последнее есть принцип переноса Нельсона [4].

УТВЕРЖДЕНИЕ 4. Система $* V$ транзитивна и замкнута относительно взятия неупорядоченных пар.

ДокАЗАТЕльство. Транзитивность $* V$ следует из определения отображения $*$. Далее, положим

$$
\begin{aligned}
& k=\max (\operatorname{rank}(x)+1, \operatorname{rank}(y)+1), \\
& \varphi(x, y) \leftrightharpoons \exists z(z=\{x, y\}) \leftrightharpoons \exists z \forall t \in z(t=x \vee t=y)
\end{aligned}
$$

Квантор существования в формуле $\varphi$ ограничен множеством $A_{k}$. Суперструктура $V$ замкнута относительно взятия неупорядоченных пар: $V \models \forall x \forall y \varphi(x, y)$. Тогда утверждение 3 дает $* V \models \forall x \forall y \varphi(x, y)$. Утверждение доказано.

Формулу $\varphi(\vec{x})$ называем абсолютной, если она абсолютна для $V$ и $* V$ [7]. Для абсолютной формулы $\varphi(\vec{x})$ ввиду (5) имеем $\varphi(\vec{a}) \leftrightarrow \varphi(* \vec{a})$. Абсолютность формул [7] $x \subset y$, $\operatorname{rel}(x), \operatorname{fnc}(x), \operatorname{trans}(x), \operatorname{ord}(x)$ тогда дает

$$
\begin{aligned}
& a \subset b \leftrightarrow * a \subset * b, \operatorname{rel}(a) \leftrightarrow \operatorname{rel}(* a), \operatorname{fnc}(a) \leftrightarrow \operatorname{fnc}(* a), \\
& \operatorname{trans}(a) \leftrightarrow \operatorname{trans}(* a), \operatorname{ord}(a) \leftrightarrow \operatorname{ord}(* a) .
\end{aligned}
$$


Операцию $O(\vec{x})=\{y \mid \varphi(\vec{x}, y)\}$, определяемую формулой $\varphi(\vec{x}, y)$, назьваем абсолютной, если она абсолютна для $V$ и $* V[7]$. Положим $* O^{V}(\vec{a})=O^{* V}(* \vec{a})$; в случае $O^{V}(\vec{a}) \in V$ это совпадает с уже определенным *-отображением (данная процедура аналогична распространению отображения * на "определимые подмножества" суперструктуры $V$, приводимому в [1]). Если $O$ абсолютна, то $* O(\vec{a})=O(* \vec{a})$. Поскольку $V$ и $* V$ транзитивны и замкнуты относительно взятия неупорядоченных пар, абсолютны операции [7] $\varnothing,\{x, y\}, x \cup y, x \cap y, x \backslash y, \bigcup x, x \times y, \operatorname{dom}(x), \operatorname{rng}(x)$. Поэтому

$$
\begin{aligned}
& * \varnothing=\varnothing, *\{a, b\}=\{* a, * b\}, \\
& *(a \cup b)=* a \cup * b, *(a \cap b)=* a \cap * b, *(a \backslash b)=* a \backslash * b, \\
& * \cup a=\cup * a, *(a \times b)=* a \times * b, \\
& * \operatorname{dom}(a)=\operatorname{dom}(* a), * \operatorname{rng}(a)=\operatorname{rng}(* a) .
\end{aligned}
$$

Отсюда, в частности, следует, что если $r$ - отношение на $a$, то $* r$-отношение на $* a$; если $f-$ функция из $a$ в $b$, то $* f-$ функция из $* a$ в $* b$, причем $*(f(a))=* f(* a)$.

Операция $\mathscr{P}$ является абсолютной для $V$. В самом деле,

$$
\mathscr{P}^{V}(a)=\{b \in V \mid b \subset a\}=\mathscr{P}(a) \cap V=\mathscr{P}(a)
$$

так как $\mathscr{P}(a) \subset V$. В то же время $\mathscr{P}$ не абсолютна для $* V$, ибо

$$
\mathscr{P}^{* V}(* f)=\mathscr{P}(* f) \cap * V \neq \mathscr{P}(* f)
$$

( “внутренняя" степень внутреннего множества $* f$ состоит из всех его внутренних подмножеств).

УТВЕРЖДЕНИЕ 5. Суперструктура $V$ удовлетворяет аксиомам $\mathrm{A} 0, \mathrm{~A} 1, \mathrm{~A} 2, \mathrm{~A} 3$, A4, A5, А8, А9. Аксиомы А6 и А7 не выполняются в $V$.

ДокАЗАТЕЛЬСтво. (А0) Ввиду абсолютности атомарных формул и $\varnothing$ имеем

$$
\begin{aligned}
V \models & (A 0) \leftrightarrow V \models \forall x(x \in S \leftrightarrow x \neq \varnothing \wedge \forall y(y \notin x)) \\
& \leftrightarrow \forall x \in V(x \in S \leftrightarrow x \neq \varnothing \wedge \forall y \in V(y \notin x)) .
\end{aligned}
$$

Последнее истинно в силу аксиомы атомов.

(A1) Ввиду абсолютности формулы $x \subset y$ имеем

$$
\begin{aligned}
V & =(\mathrm{A} 1) \leftrightarrow V \models \forall X \forall Y(X \subset Y \wedge Y \subset X \rightarrow X=Y) \\
& \leftrightarrow \forall X \in V \forall Y \in V(X \subset Y \wedge Y \subset X \rightarrow X=Y) .
\end{aligned}
$$

Последнее истинно в силу аксиомы объемности.

(A2) Очевидно, так как $V$ замкнуто относительно взятия неупорядоченньх пар.

(А3) Имеем

$$
\begin{aligned}
V & \models(\mathrm{A} 3) \leftrightarrow V \models \forall \vec{y} \forall X \exists Y(Y=\{x \in X \mid \varphi(x, \vec{y})\}) \\
& \leftrightarrow \forall \vec{y} \in V \forall X \in V \exists Y \in V(Y=\{x \in V \mid x \in X \wedge V \models \varphi(x, \vec{y})\}) \\
& \leftrightarrow \forall \vec{y} \in V \forall X \in V \exists Y \in V(Y=\{x \in X|V|=(x, \vec{y})\}) .
\end{aligned}
$$


Последнее получено с учетом транзитивности $V$. Множество $Y$ в (8) есть подмножество множества $X$. Если $X \in V$, то $X \subset V_{n}$ для некоторого $n \in \omega$; тогда $Y \subset V_{n}$ и $Y \in V$. Тем самьм (8) истинно.

(А4) Ввиду абсолютности операции $\cup$ имеем

$$
\begin{aligned}
V \models & (\mathrm{A} 4) \leftrightarrow V \models \forall X \exists Y(Y=\cup X) \\
& \leftrightarrow \forall X \in V \exists Y \in V(Y=\cup X) .
\end{aligned}
$$

Если $X \in V_{1}$, то $Y=\cup X=\varnothing \in V$. Если $X \in V_{n}, n \geqslant 2$, то $Y \in V_{n-1}$, и потому $Y \in V$. Тем самьм (9) истинно.

(А5) Ввиду абсолютности для $V$ операции $\mathscr{P}$ имеем

$$
\begin{aligned}
V & =(\mathrm{A} 5) \leftrightarrow V \models \forall X \exists Y(Y=\mathscr{P}(X)) \\
& \leftrightarrow \forall X \in V \exists Y \in V(Y=\mathscr{P}(X)) .
\end{aligned}
$$

Последнее истинно.

(А6) Поскольку $S$ бесконечно, можно считать, что $\omega \subset S$, так что $\omega \in V$. Однако последовательность (2) - функциональный образ $\omega$ - не является элементом $V$.

(А7) Не выполняется в $V$ очевидньп образом.

(А8) Так как формула $\exists x \in X(x \cap X=\varnothing)$ абсолютна, то

$$
V \models(\mathrm{A} 8) \leftrightarrow \forall X \in V(X \neq \varnothing \rightarrow \exists x \in X(x \cap X=\varnothing))
$$

Последнее истинно в силу аксиомы регулярности.

(A9) Ввиду абсолютности fnc $(x)$ и $\operatorname{dom}(x)$ имеем

$$
\begin{aligned}
V & =(\mathrm{A} 9) \leftrightarrow \forall X \in V(X \neq \varnothing \wedge X \cap S=\varnothing \wedge \forall Y \in X(Y \neq \varnothing) \\
& \rightarrow \exists f \in V(\operatorname{fnc}(f) \wedge \operatorname{dom}(f)=X \wedge \forall Y \in X(f(Y) \in Y))) .
\end{aligned}
$$

Последнее истинно. В самом деле, пусть $X \in V_{n}, n \geqslant 2$, и $f$ есть функция выбора на $X$. Тогда $f=\{(Y, f(Y)) \mid Y \in X\} \in V_{n+2}$, так что $f \in V$.

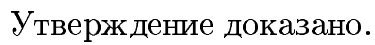

Согласно утверждению 3 выражения (6)-(12) могут быть перенесены в $* V$ (кванторы сушествования в (8)-(10), (12) ограничены соответствуюшим уровнем суперструктуры $V)$. Утверждение 4 позволяет добавить аксиому пары. В результате получаем

$$
\begin{aligned}
& *(\mathrm{~A} 0): \forall x \in * V(x \in * S \leftrightarrow x \neq \varnothing \wedge \forall y \in * V(y \notin x)), \\
& *(\mathrm{~A} 1): \forall X \in * V \forall Y \in * V(X \subset Y \wedge Y \subset X \rightarrow X=Y), \\
& *(\mathrm{~A} 2): \forall x \in * V \forall y \in * V(\{x, y\} \in * V), \\
& *(\mathrm{~A} 3): \forall \vec{y} \in * V \forall X \in * V(\{x \in X|* V|=\varphi(x, \vec{y})\} \in * V), \\
& *(\mathrm{~A} 4): \forall X \in * V(\cup X \in * V), \\
& *(\mathrm{~A} 5): \forall X \in * V(\mathscr{P}(X) \cap * V \in * V), \\
& *(\mathrm{~A} 8): \forall X \in * V(X \neq \varnothing \rightarrow \exists x \in X(x \cap X=\varnothing)), \\
& *(\mathrm{~A} 9): \forall X \in * V(X \neq \varnothing \wedge X \cap * S=\varnothing \wedge \forall Y \in X(Y \neq \varnothing) \\
& \rightarrow \rightarrow \exists f \in * V(f-\text { функция выбора на } X)) .
\end{aligned}
$$


Выражения $*(\mathrm{~A} 0)-*(\mathrm{~A} 5), *(\mathrm{~A} 8), *(\mathrm{~A} 9)$ играют роль теоретико-множественных аксиом для нестандартной системы $* V$. Отметим, что важный для приложений принцип внутреннего определения (или теорема о внутренних множествах) $[1$, с. 69] есть в данном случае просто аксиома $*($ A3) (ср. также с [2, с. 33]).

Данная работа посвящается памяти Вячеслава Федоровича Яковлева.

\section{СПИСОК ЦИТИРОВАННОЙ ЛИТЕРАТУРЫ}

[1] Девис М. Прикладной нестандартный анализ. М.: Мир, 1980.

[2] Альбеверио С., Фенстад Й., Хеэг-Крон Р., Линдстрем Т. Нестандартные методы в стохастическом анализе и математической физике. М.: Мир, 1990.

[3] Loeb P., Wolff M. P.H. (ed. ) Nonstandard Analysis for the Working Mathematician. Dordrecht: Kluwer, 2000.

[4] Nelson E. Internal set theory: a new approach to nonstandard analysis // Bull. Amer. Math. Soc. 1977. V. 83. №6. P. 1165-1198.

[5] Гордон Е.И., Кусраев А. Г., Кутателадзе С. С. Инфиниитезимальный анализ. Ч. 1. Новосибирск: Изд-во ИМ, 2001.

[6] Яковлев В.Ф. Расширенный универсум для систем с минимальными структурами // Докл. AH CCCP. 1991. Т. 319. №1. С. 103-105.

[7] Йех Т. Теория множеств и метод форсинга. М.: Мир, 1973.

[8] Кейслер Г., Чэн Ч. Ч. Теория моделей. М.: Мир, 1977.

[9] Маккаи М. Допустимые множества и бесконечная логика // Справочная книга по математической логике. Ч. 1. М.: Наука, 1982. С. 235-288. 